Supplement of Earth Syst. Dynam. Discuss., 6, 1999-2042, 2015

http://www.earth-syst-dynam-discuss.net/6/1999/2015/

doi:10.5194/esdd-6-1999-2015-supplement

(C) Author(s) 2015. CC Attribution 3.0 License.

(c) (i)

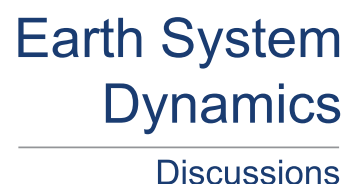

Discussions

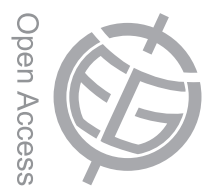

Supplement of

\title{
A novel bias correction methodology for climate impact simulations
}

\section{S. Sippel et al.}

Correspondence to: S. Sippel (ssippel@bgc-jena.mpg.de)

The copyright of individual parts of the supplement might differ from the CC-BY 3.0 licence. 

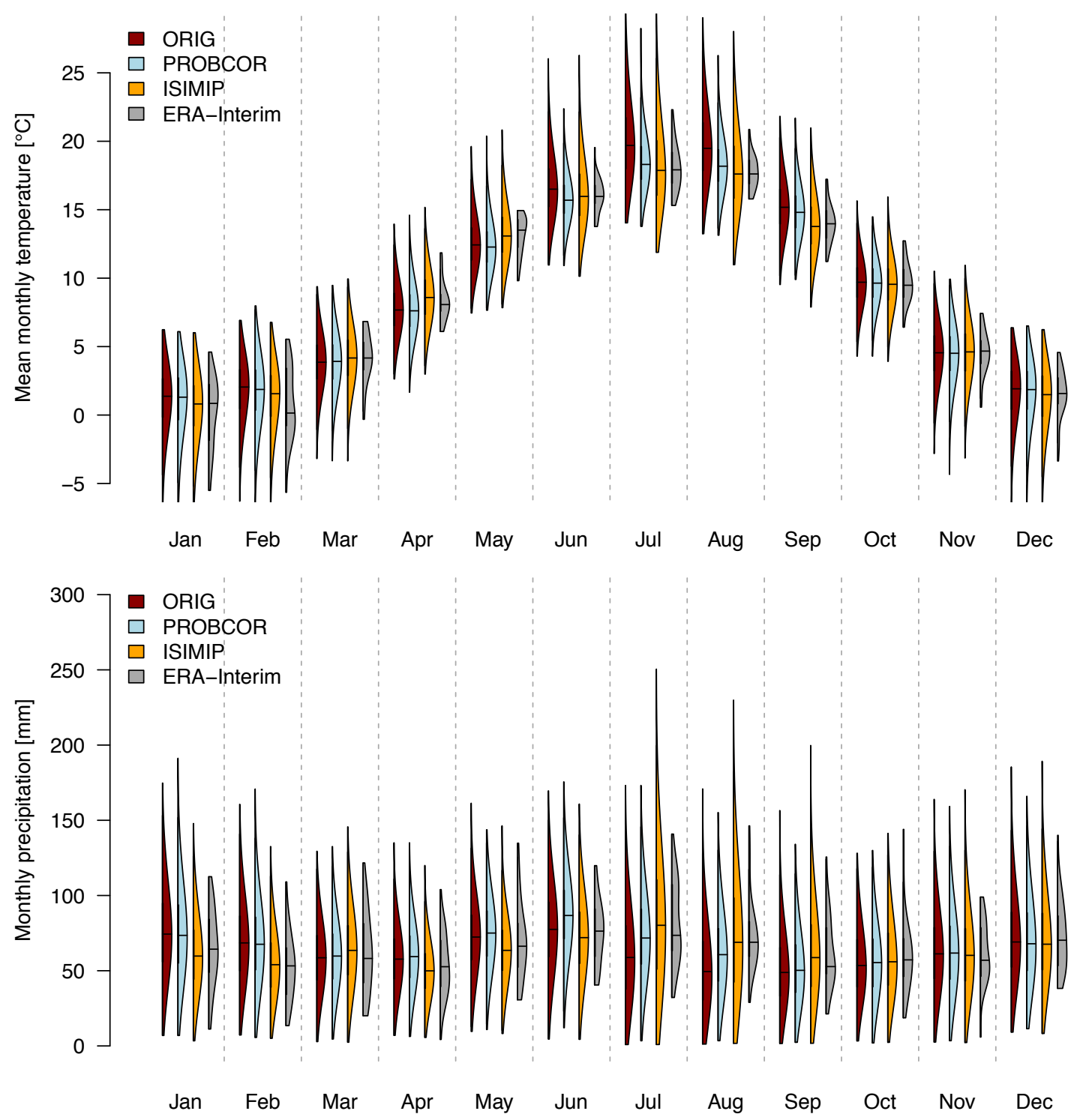

Figure S1: Evaluation of bias-adjustment at the monthly time scale for temperature (top panel) and precipitation (bottom panel) in Central Europe. Virtually no changes are induced by the probabilistic resampling scheme in non-summer months, whereas an additive (temperature) or mutliplicative (precipitation) of monthly means might result in relatively pronounced changes in higher statistical moments of the bias-adjusted distribution depending of the magnitude of the bias in monthly means. 

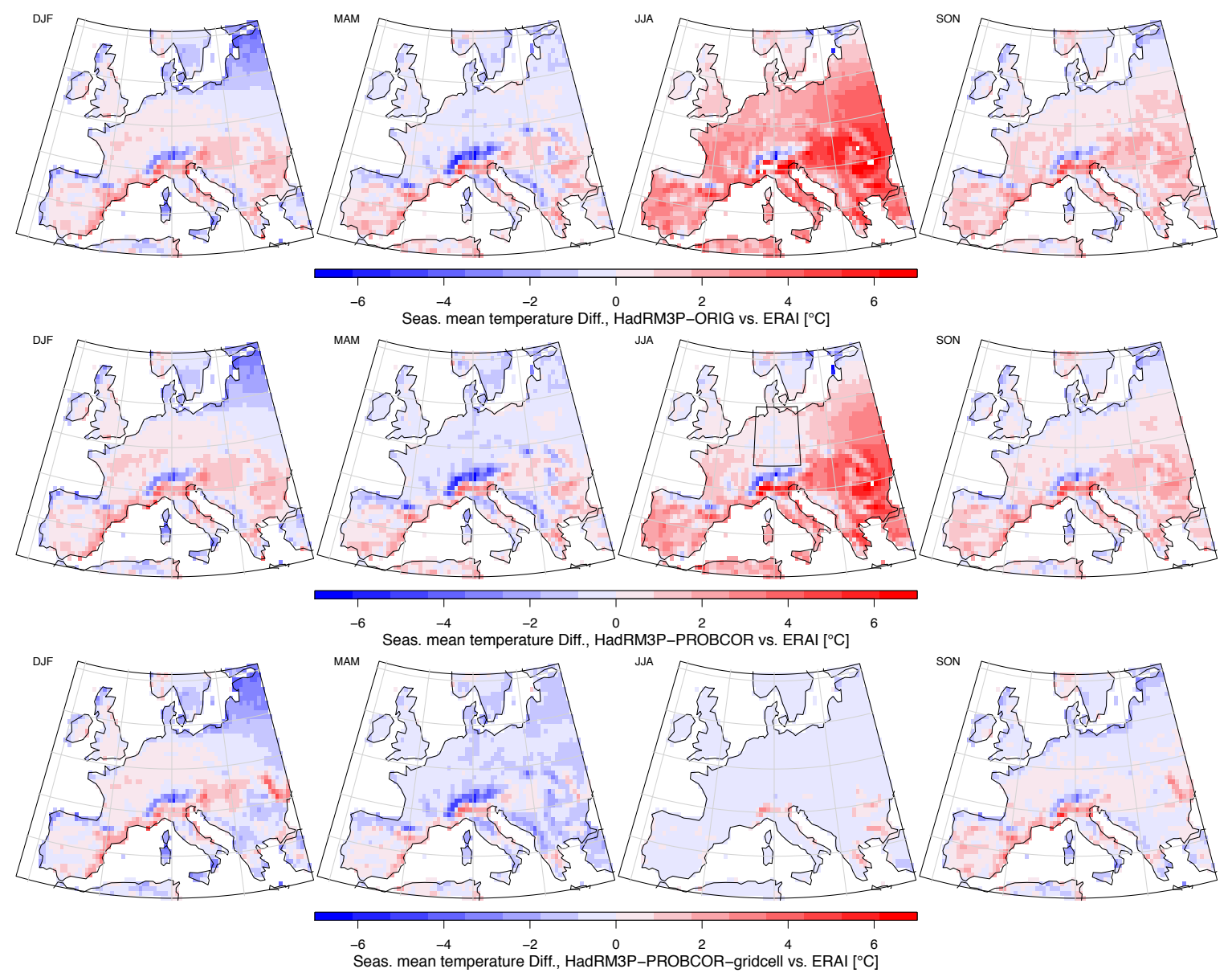

Figure S2a: Biases in the HadRM3P simulated distribution of seasonal mean temperatures: Deviation in the ensemble mean w.r.t. the ERA-Interim dataset, (top) in the original HadRM3P simulated distribution; (middle) in the resampled ensemble using a Central Europe average JJA mean temperature constraint; (bottom) in a resampled ensemble using a JJA mean temperature constraint on each grid cell. 


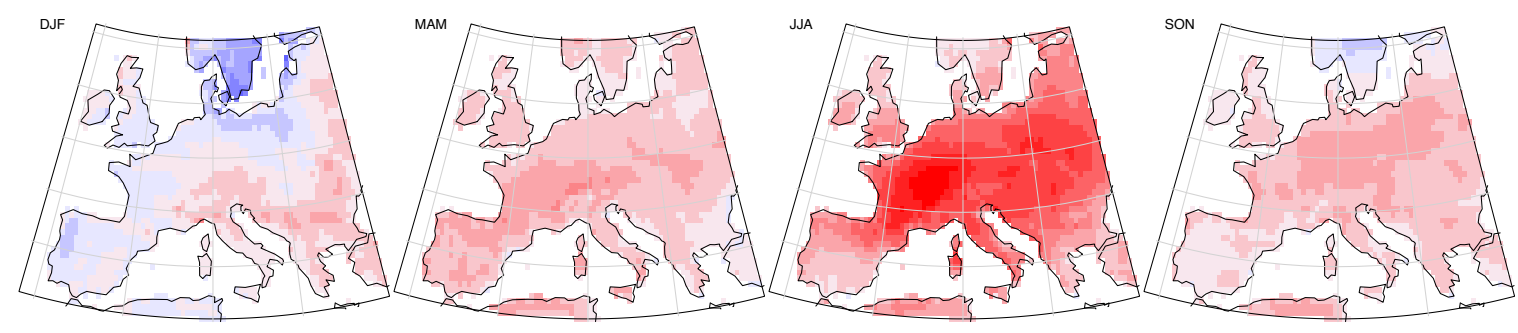

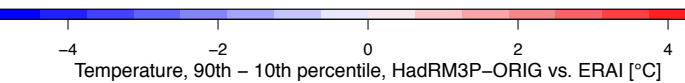
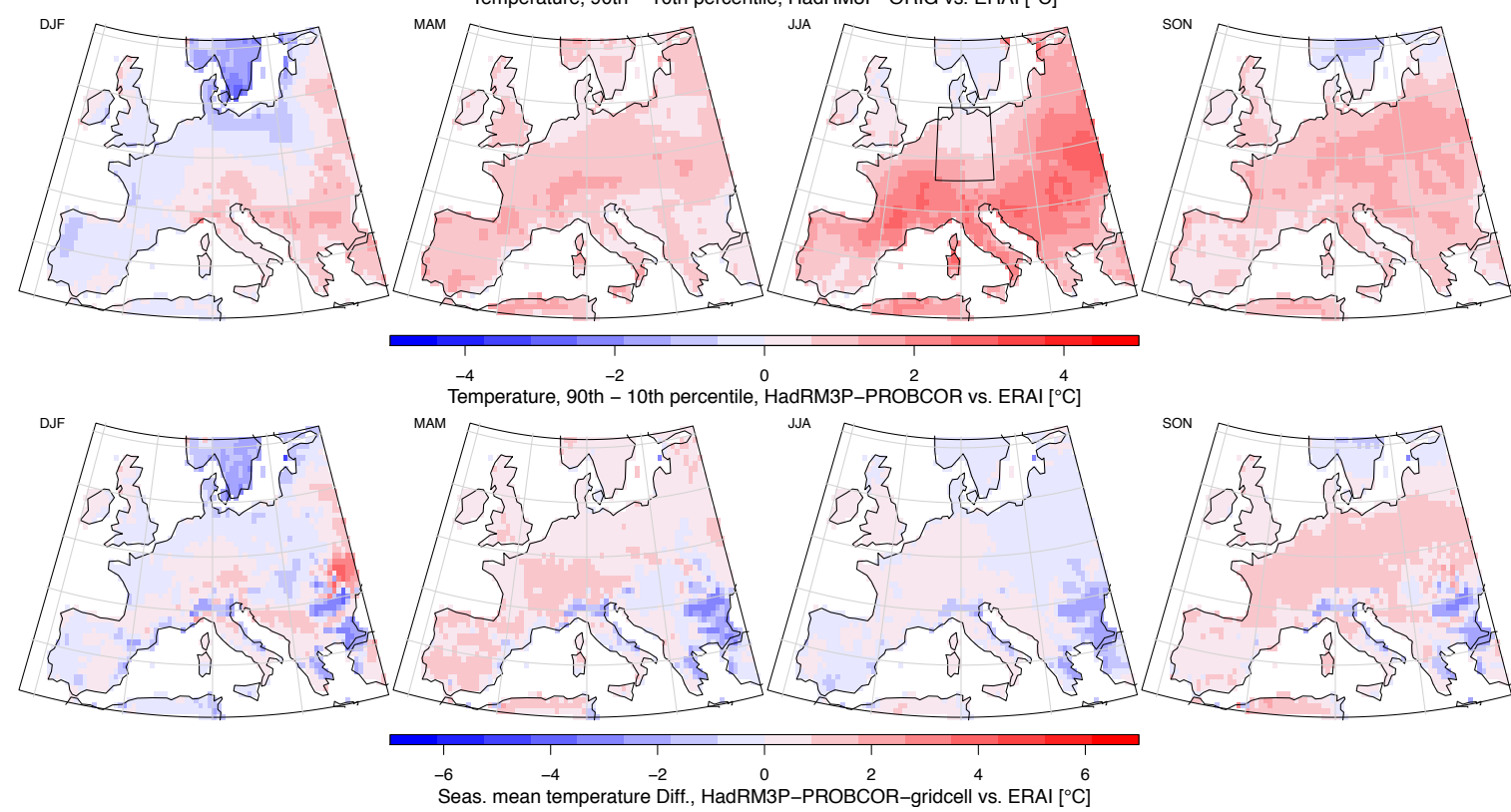

Figure S2b: Biases in the HadRM3P simulated distribution of seasonal mean temperatures: Deviation in the interdecile range w.r.t. the ERA-Interim dataset, (top) in the original HadRM3P simulated distribution; (middle) in the resampled ensemble using a Central Europe average JJA mean temperature constraint; (bottom) in a resampled ensemble using a JJA mean temperature constraint on each grid cell. 

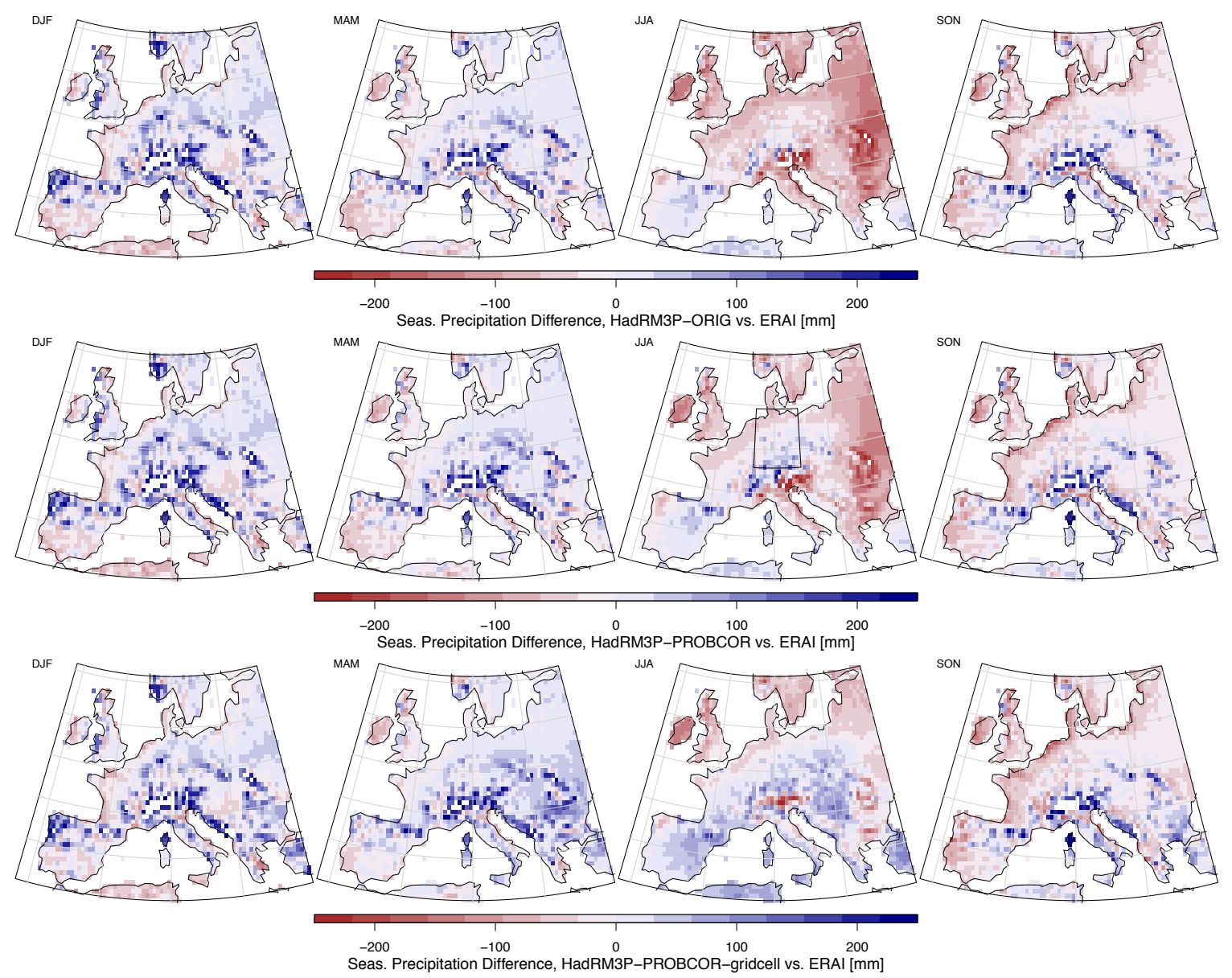

Figure S3a: Biases in the HadRM3P simulated distribution of seasonal mean precipitation: Deviation in the ensemble mean w.r.t. the ERA-Interim dataset, (top) in the original HadRM3P simulated distribution; (middle) in the resampled ensemble using a Central Europe average JJA mean temperature constraint; (bottom) in a resampled ensemble using a JJA mean temperature constraint on each grid cell. 

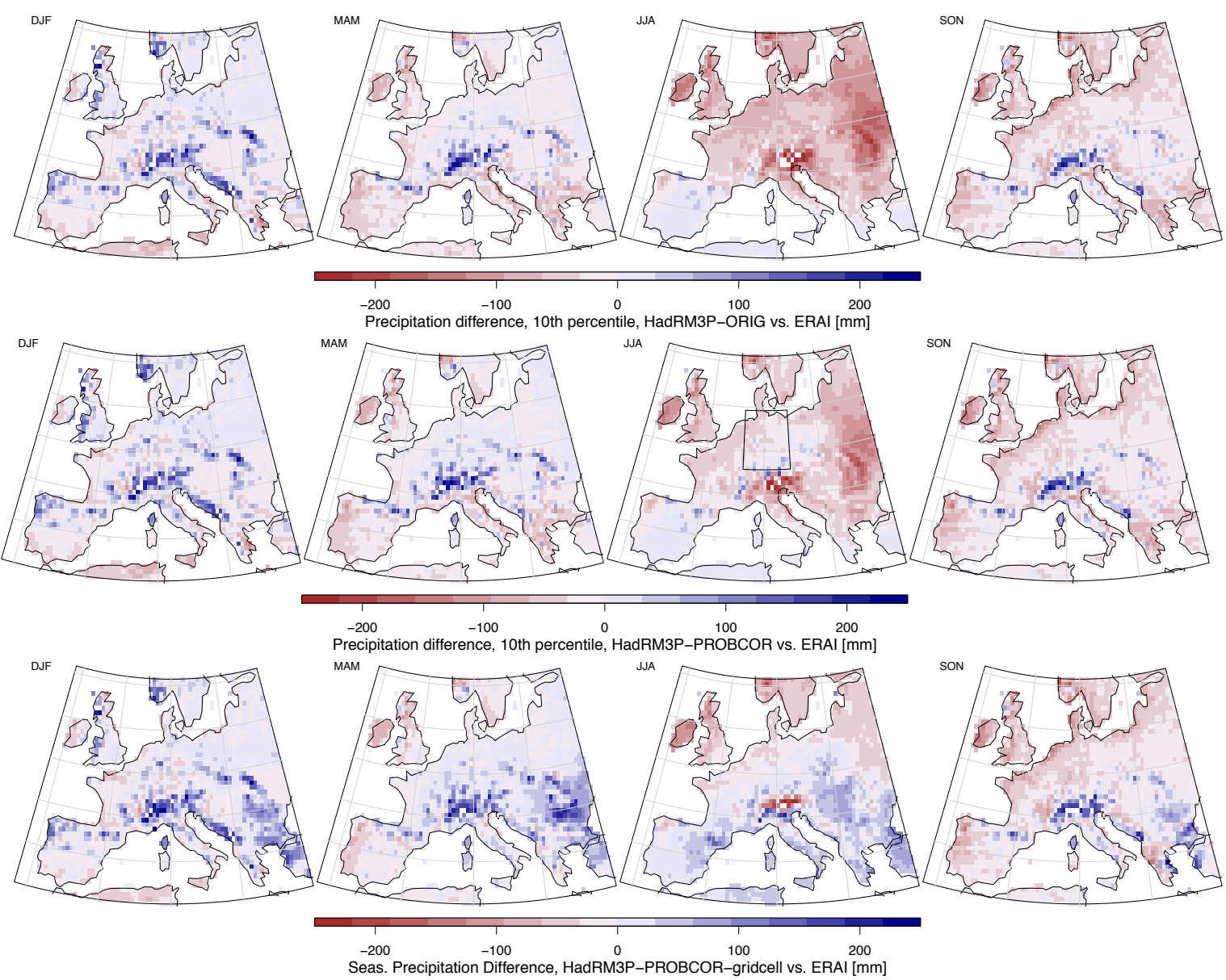

Seas. Precipitation Difference, HadRM3P-PROBCOR-gridcell vs. ERAI [mm]

Figure S3b: Biases in the HadRM3P simulated distribution of the dry tail of seasonal mean precipitation

(10th percentile): Deviation in the 10th percentile w.r.t. the ERA-Interim dataset, (top) in the original

HadRM3P simulated distribution; (middle) in the resampled ensemble using a Central Europe average JJA mean temperature constraint; (bottom) in a resampled ensemble using a JJA mean temperature constraint on each grid cell. 
a

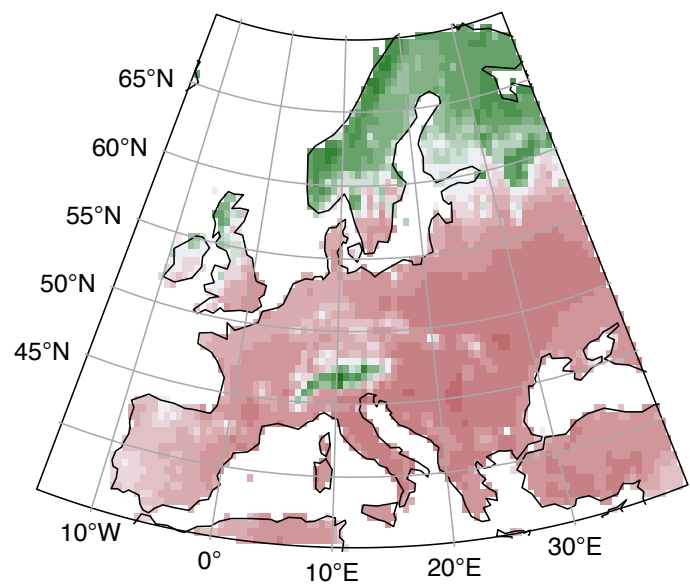

c

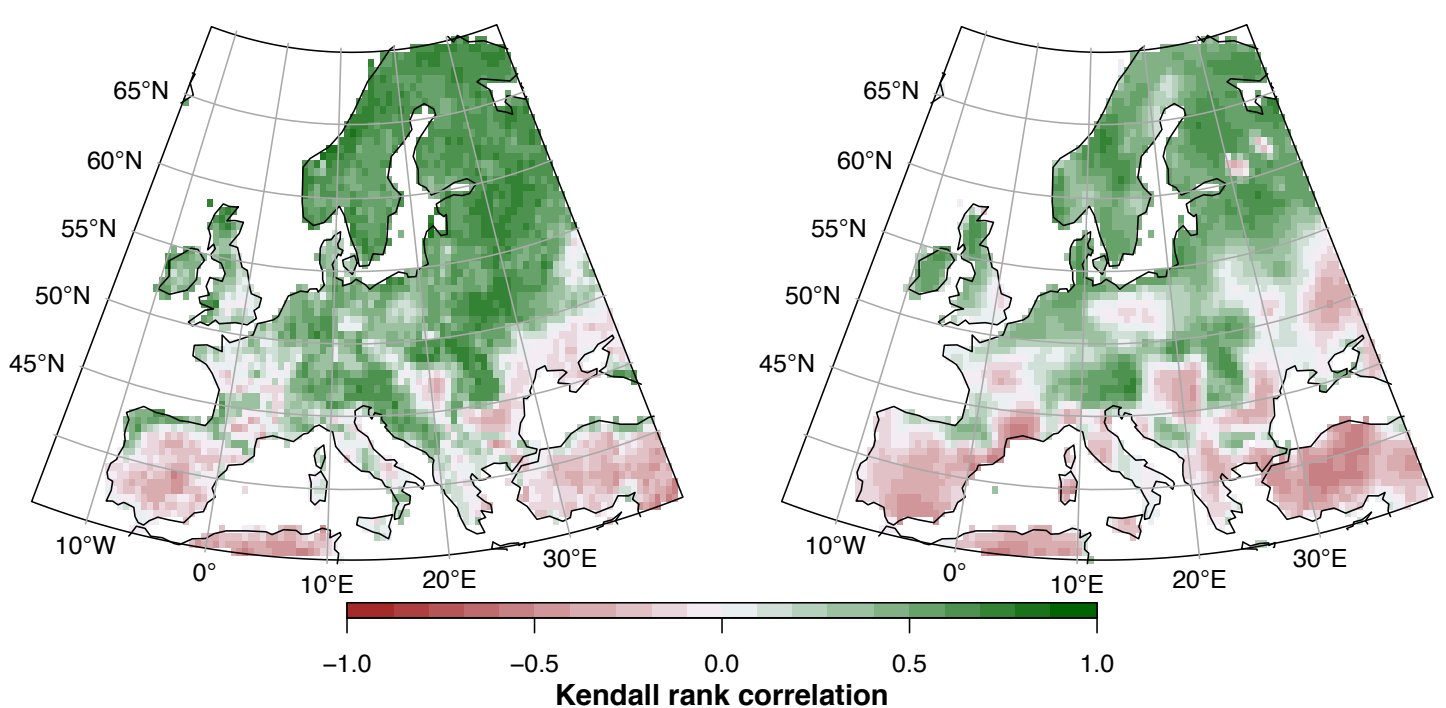

b HadRM3P - bias. cor.

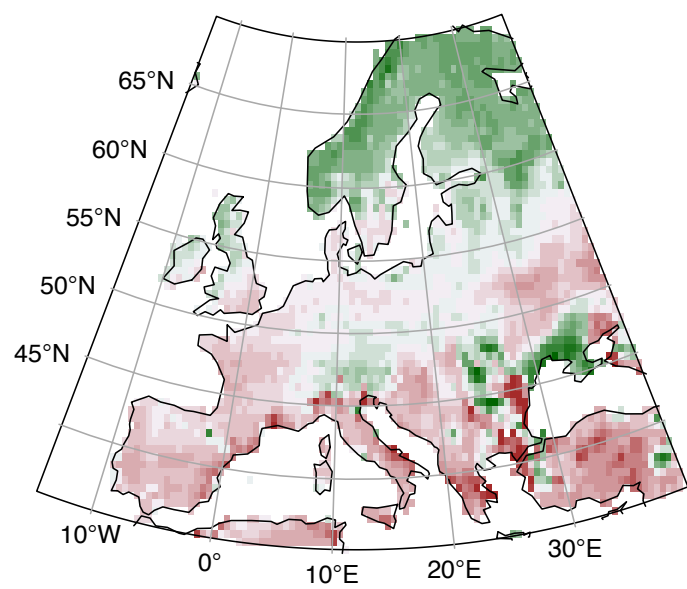

d $\quad$ ERA-Interim

Figure S4: Land-astmosphere coupling in the original model ensemble (a), in the resampled 'bias-corrected' ensemble (b), in an upscaled dataset of latent heat fluxes (c), and in the ERA-Interim reanalysis dataset. All plots show the Kendall rank correlation coefficient between mean summer temperatures (JJA) and mean summer latent heat fluxes as a robust measure for the land-atmosphere coupling strength. 


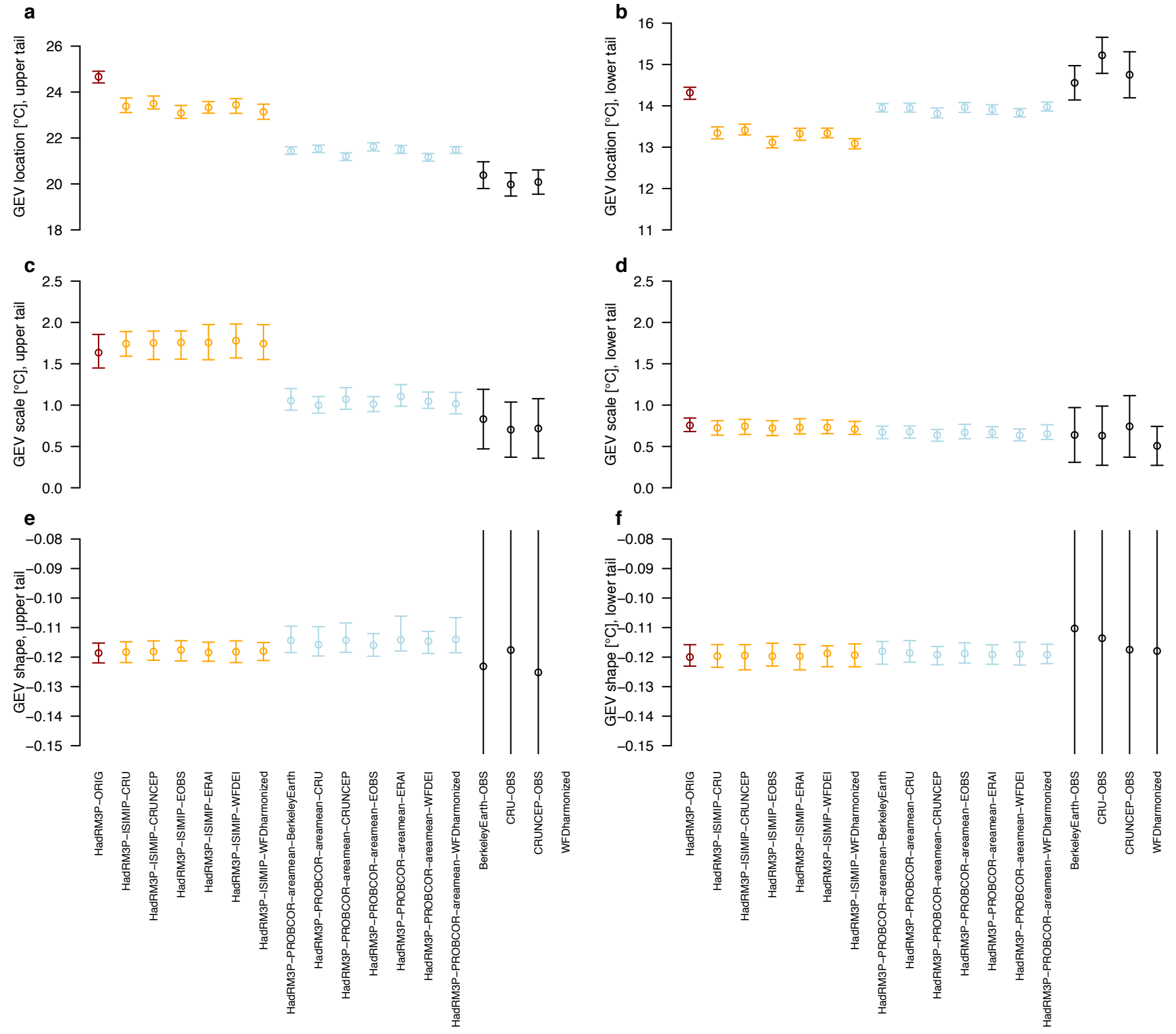

Figure S5a: Extreme value analysis of monthly temperatures (JJA) derived from bias-corrected ensemble simulations as spatial averages over Central Europe. Location, scale and shape parameters (top, middle, bottom panel, respectively) derived from Generalized Extreme Value distributions fitted to the upper and lower tail (right and left, respectively) of monthly simulations. 


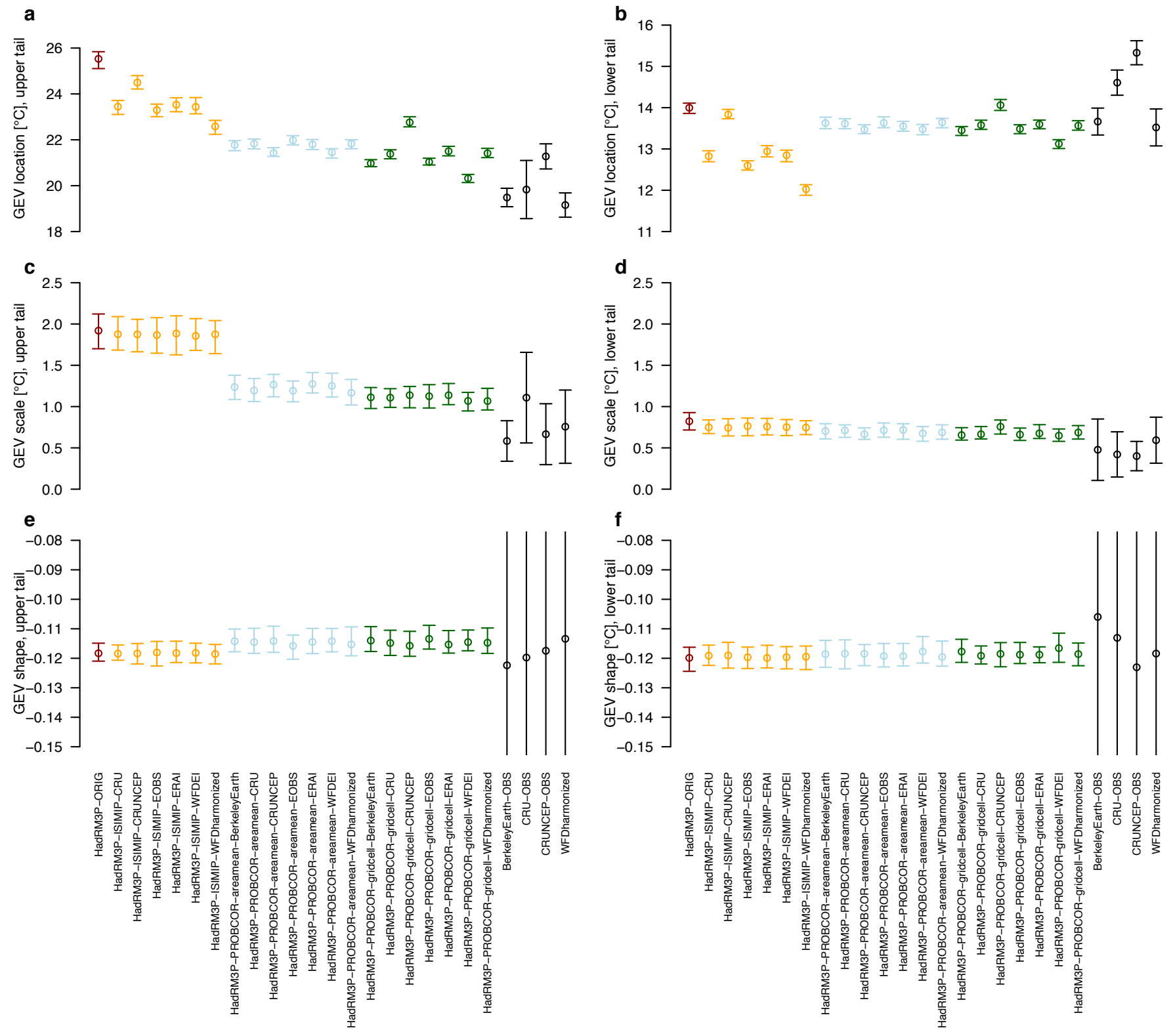

Figure S5b: Extreme value analysis of monthly temperatures (JJA) derived from bias-corrected ensemble simulations for an individual grid cell ('Jena pixel'). Location, scale and shape parameters (top, middle, bottom panel, respectively) derived from Generalized Extreme Value distributions fitted to the upper and lower tail (right and left, respectively) of monthly simulations. 

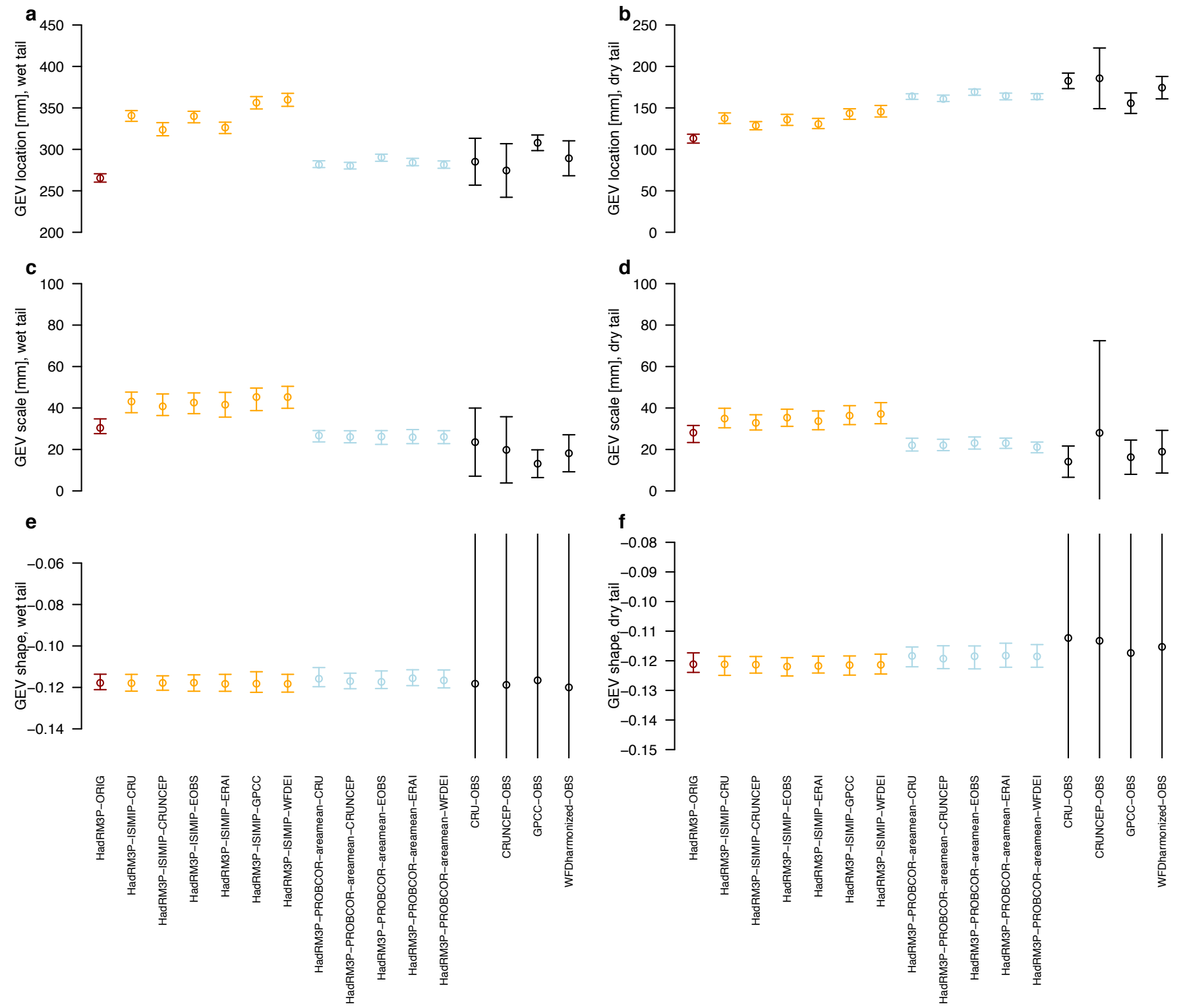

Figure S6a: Extreme value analysis of seasonal precipitation (JJA) derived from bias-corrected ensemble simulations as spatial averages over Central Europe. Location, scale and shape parameters (top, middle, bottom panel, respectively) derived from Generalized Extreme Value distributions fitted to the upper and lower tail (right and left, respectively) of monthly simulations. 

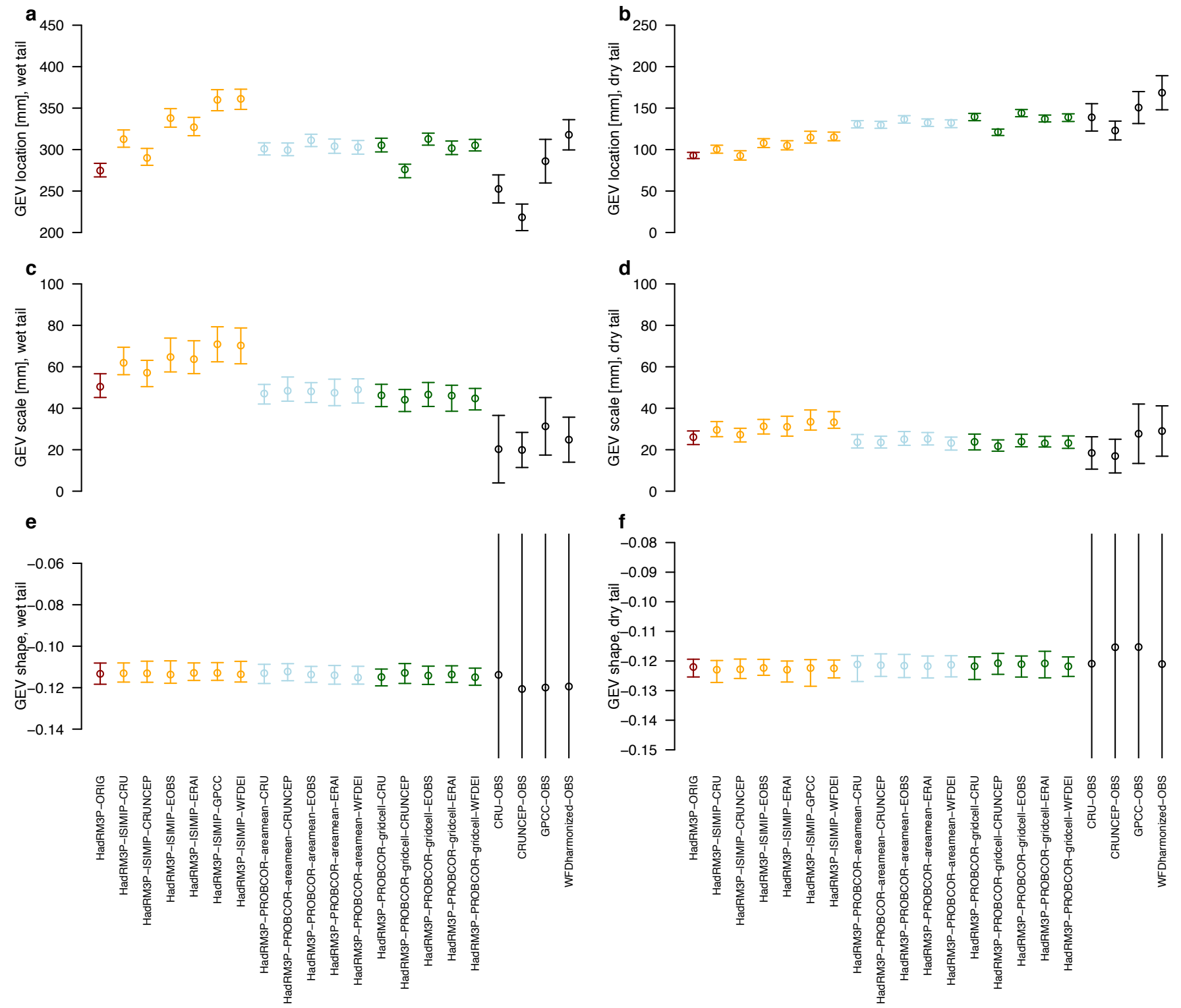

Figure S6b: Extreme value analysis of seasonal precipitation (JJA) derived from bias-corrected ensemble simulations for an individual grid cell ('Jena pixel'). Location, scale and shape parameters (top, middle, bottom panel, respectively) derived from Generalized Extreme Value distributions fitted to the upper and lower tail (right and left, respectively) of monthly simulations. 